the education and training of the staff who will be involved in cytotoxic reconstitution which is followed by an invaluable chapter on the management of extrasavation of cytotoxic drugs.

Section two of the book is likely to be a constant source of reference. It comprises a valuable alphabetical listing of monograhs of individual drugs that are used intravenously. Each monograph has been prepared by a named member of the working group according to a common structure. There is a section on nomenclature which includes the names of manufacturers and suppliers in the UK. There follows sections on chemistry, stability profile, clinical usage, details of how individual drugs are prepared for injection or infusion

\section{Evolution of Cancer}

S. Okuyama and H. Mishina, Tokyo: University of Tokyo Press, 1990, 266 pp. £57.50.

This is a highly provocative book, written by two clinical radiotherapists, on the evolution of cancer. The authors base their approach to the treatment of cancer on the hypothesis that cancer represents a process of evolution in reverse, which they call 'devolution'. This concept is applied to develop theories on carcinogenesis, epidemiology, diagnosis and especially the strategy of cancer treatment. Fanconi's anaemia is cited as an evolutionary experiment of nature on carcinogenesis. The tumours described in patients with the DNA repair syndrome were classified according to their epithelial, -non-epithelial and gonadal origin. The early manifestation of non-malignancies and the later occurrence of epithelial tumours is considered as a support for the evolution theory. The cancer incidence in Japan following the explosion of the atomic bombs would also support this evolution theory. The parallel evolution of biology with the

An Introduction to Radiobiology, A.H.W. Nias, New York: John Wiley \& Sons, 1990, 346 pp. £17.50.

The stated aim of this book is 'to provide an extremely readable introduction to the subject of radiobiology'. However, it is perhaps better described as an introductory textbook of cellular radiobiology designed for would-be radiobiologists and medical physicists to peruse prior to consulting more advanced sources. It is not a primer to be read by the uninitiated where the reader is gently led to deeper levels of understanding. Despite this caveat the book has many admirable qualities in its 346 pages including copious graphics and illustrative diagrams and succinct summaries of the basic conclusions at the end of each of its 20 chapters.

Chapter 1 is a rather eclectic ten pages of the history of radiobiology with paragraphs on radiation, radiation units and timescales. Chapter 2, although entitled 'Cells and Tissues' is really devoted to a single aspect of cell biology viz. cell synchronisation and methods to attain it. Unfortunately, the first sentence of this chapter implies that all human cells are nucleate and this is only amended later in the text. The author also repeats the ancient phrases about $G_{1}$ and $G_{2}$ being 'gaps in our knowledge' of cell cycle events. This is hardly tenable after nearly $\mathbf{4 0}$ years of research into pre- and post-DNA phase biochemistry and cell metabolism. Chapter 3 goes into the greater details of cell population kinetics.

Chapter 4 is the standard introductory account of radiation physics to be found in numerous kindred textbooks. It is high time these were all updated to include the last decades exciting advances in microdosimetry so as to illustrate the meaning of 'dose' at the cellular, subcellular and molecular levels.

Chapter 5 'Subcellular Radiobiology' is only 14 pages and purports to cover 'radiation biochemistry', 'molecular radiobiology' and 'cytogenetics' - a very tall order. A critical opportunity has however been missed, namely the provision together with handling precautions. The final section considers how residual drug and contaminated articles should be destroyed. It should be emphasised that the working group has deliberately refrained from providing details of 'standard protocols for various indications'.

In summary this is a book that should be read by any members of the 'Oncology Team' involved in setting up a reconstitution service. It should also be available in the departmental library of all Oncology Departments and all Pharmacies where any cytotoxic drugs are reconstituted and administered.

T.J. Perren

physicochemical alterations on earth are also cited in support of the evolution theory of cancer. Such arguments are then used for the formulation of a therapeutic strategy, starting with the repair of DNA damage, via carbohydrate biochemistry to immunotherapy and antiviral therapy.

A large variety of arguments are used to formulate a strategy in surgery, radiotherapy, chemotherapy and immunotherapy. For most readers of this book it will be difficult to find practical advice regarding strategy of treatment that will have to be used. The two last chapters deal with differentiation of cancer cells induced by the immunostimulant Bestatin, sex hormones and prostaglandins.

This book is difficult reading, because the authors use a wealth of biological data to search for support of their concepts on evolution and devolution of cancer. They have not succeeded in formulating a clear strategy for treatment, based on the postulated mechanisms of evolution.

F.J. Cleton

of a clear popular exposition of DNA damage and its repair and misrepair. This topic, so central to the chain of events between energy absorption and final expression of biological damage, is barely mentioned. This contrasts with repair at the cellular and tissue levels which are fully discussed in Chapter 7.

Chapter 6 describes the post irradiation fate of individual cells - giant cell formation, interphase death and then focusses most of its attention, quite correctly, on mitotic death.

Chapter 8 on 'Intrinsic Radiosensitivity' discusses the various shapes of cell survival curves using both in vitro and in vivo studies. It touches on the 'SF at 2 Gy' a current hot topic in radiobiology but much of the chapter hinges on the older and all but discredited target theories, rather than more recent interpretations of repair/saturation models. Two small errors have also crept into the algebra of this chapter. The next section is on the special qualities of densely ionising radiations such as alpha particles, neutrons and pi-mesons.

Chapter 10, on the radiosensitising and dose modifying effects of oxygen is good but marred by one of the figures (Figure 10:7) which indicates a crossing over of two survival curves clearly implying oxygen protects cells at doses below $2 \mathrm{~Gy}$. This contradicts the text and will puzzle all casual readers. The next chapter is on sulphydryl(-SH) containing radioprotective agents and on electron affinic radiosensitisers and is illustrated by data on clonogenic assays.

Chapter 12 compares the radiobiology of normal and malignant cells with the emphasis yet again on clonogenic assays. The book is beginning to feel repetitive. There follow two chapters of pathology, the first on tissue effects and the second on the whole body radiation syndromes.

Chapters 15 and 16 are a potted version of the radiobiological basis of fractionation radiotherapy.

The author describes the final 60 pages as covering the effects of low dose and low dose rate radiation; the study of 
which will allow us to devise 'guidelines for the safe use of radiation in everyday life'. This laudable intention has, however, not been achieved. The major health hazards of low dose radiation are cancer induction, genetic damage and to a lesser extent CNS damage following irradiation of the embryo and foetus. But these receive scant attention, with just two pages each on the genetic and foetal effects and only nine pages on carcinogenesis.

The perfect textbook has never been written; but a sub-

\section{Neoadjuvant Chemotherapy in Invasive Bladder Cancer}

Edited by Ted A.W. Splinter and Howard I. Scher, New York: Willey-Liss, 1990, 241 pp. £57.50.

It is now 7 years since the demonstration by Carmichael in 1983 that methotrexate combined with cisplatin produced durable complete remission in a small proportion of patients with measurable metastatic bladder cancer. Since this time the four drug combination developed by the Memorial Hospital has become the most widely used regimen for combination, because of suggestion that there was a higher complete response rate using this regimen in patients with metastases. As a consequence of these observations, today the majority of centres involved in research in bladder cancer treatment have now taken this observation and use platinum containing combination instead of radiation as their standard adjuvant regimen for the treatment of invasive bladder tumours without metastases. This book which is the proceedings of a workshop held in San Francisco 18 months ago proves to be an invaluable resource of reference to the effect of this change in policy, though sadly it raises as many questions as it answers.

After a series of review papers about chemotherapy, radiation, pathology of tumours after treatment and trial design, there are five papers about randomised trials and nine about phase 2 studies from 12 different countries and six chapters about assessing response to treatment. Sadly in the critical area of the randomised trial none have advanced to the stage of having any information on response or survival. Despite the fact that it is going to be at least another 2-3 years before information will emerge from the randomised trials, the maturation of the phase 2 studies which led to the initial enthusiasm for this approach and the increase in number of other authors who have got phase 2 data, does make it easier to get a feel that the likely benefits are more likely to be closer to those seen from using chemotherapy to treat primary head and neck cancer than from that gained from using it as adjuvant for breast cancer.

The critical information is that provided in the first chapter by Howard Scher who has done an excellent work in summarising the literature and the contributions from participants. This shows clearly that the proportion of patients with previously untreated primary bladder cancer achieving complete remission while only being $11 \%$ for single agent platinum, was more than $20 \%$ for all platinum based

\section{Current Genitourinary Cancer Surgery}

Edited by E. Crawford and S. Das, London: Lea \& Febiger, 1990, 249 pp. $£ 87.35$.

Professor Crawford and Dr Das have persuaded 81 surgeons and physicians to contribute to this edition of Genitourinary Cancer Surgery. No significant tumour or tumour-type has been ignored and all are considered in a most practical way which allows both the trainee and the expert to feel at home with, and to benefit from, the views expressed. Each major section has an overview which clearly sets out the accepted basic information, draws attention to contentious issues and highlights some of the problems which both physician and surgeon must face on a daily basis. It is a book for surgeons by surgeons and very properly gives over the majority of its stantive criticism of this basic book on radiobiology must be that the balance of information is awry. There is too much stress on cellular effects, too little stress on DNA damage and much too little on the societally important low level radiation health effects of carcinogenesis, teratogenesis and genetic damage.

\section{J.E. Coggle}

combinations. However there was no difference between the two drug combinations methotrexate cisplatin $(33 \%)$ and the four drug Memorial M-VAC regimen (32\%). Though there was mention of one small trial where the two have been compared, this is obviously an important area that will need focusing on in the future, particularly as this complete remission rate is lower than that reported by many authors who have used the considerably less toxic radiation as primary treatment.

A final area of considerable controversy, which is the subject of one chapter by Reg Hall, is the issue of whether transurethral resection of tumour (TURT) should be used to reduce tumour bulk prior to chemotherapy treatment. Sadly it is rather difficult to assess his extremely important data as the text says that $70-80 \%$ of his patients remain tumour free while the data presented in the table shows that at 2 years only $50 \%$ have a bladder free of tumour following TURT and combination chemotherapy which is worse than he achieved using single agent methotrexate alone after TURT (57\% at 3 years). As none of the other authors give any information about how much resection of tumour they did prior to treatment, it is clear that more assessment of this variable is required. Given the anxiety raised from studies in prostate cancer which suggest that there may be increased intravascular and lymphatic dissemination after transurethral resection, it would be important to exclude such as effect happening in bladder cancer patients who have TURT.

The final section of the book on assessing response is very useful in its own right, because it provides important prognostic information (in particular the influence of tumour size and response to treatment on survival) as well as publishing two of the first papers that have actually gone to the trouble of computing tumour volume before and after treatment, though the figure in Sager's paper showing shrinkage of what is labelled as the uterus after chemotherapy does emphasise the limits of the technology at present.

I am sure that there can be little doubt that this book will be an important reference for the future, though its message to me is that we still have far to go if we are to improve on the figure of $30 \%$ 5-year survival for T3 bladder cancer which has been with us since the advent of megavoltage radiotherapy.

R.T.D. Oliver

test to a consideration of the indications for, and alternatives in techniques of, urological cancer surgery. Despite the number of authors, the editing has produced a marvellously uniform text of great clarity with a wealth of illustrations of the very highest quality drawing attention to details of critical surgical importance.

The editors have cast their net widely and have incorporated every relevant surgical advance without endeavouring to force the acceptance of any particular option considered.

The reader from Europe will notice the American usage of English and will be aware from such statements as 'approximately 47,100 new cases of bladder carcinoma will be diagnosed this year'; and '. . . cystectomy remains the therapeutic choice for patients with invasive bladder cancer, that this book is primarily written for Americans. The case for surgi- 